\title{
Comparison of Two Different Intravitreal Injection Techniques
}

\author{
Harvey S Uy (D) ${ }^{1-3}$ \\ Jose Carlo M Artiaga (iD) ${ }^{4}$ \\ 'Department of Ophthalmology and \\ Visual Sciences, University of the \\ Philippines, Manila, Philippines; ${ }^{2}$ Peregrine \\ Eye and Laser Institute, Makati City, \\ Philippines; ${ }^{3}$ St. Luke's Medical Center, \\ Quezon City, Philippines; ${ }^{4}$ St. Luke's \\ Medical Center Global City, Taguig City, \\ Philippines
}

Objective: To compare the effectiveness, procedure time and safety outcomes of two different intravitreal injections (IVI) techniques.

Methods: This was a prospective, single-center, randomized clinical trial of 200 adult eyes receiving intravitreal medications for various indications. Eyes were assigned (1:1) to undergo IVI using either an intravitreal injection guide (IIG) $(n=100)$ or conventional dual blade speculum plus surgical caliper (DBS) $(n=100)$. All IVI were administered using a 30-gauge needle placed $4 \mathrm{~mm}$ posterior to the inferior limbus. The main outcome measures were rate of successful IVI administration, procedure time (seconds) as measured by a stopwatch from application to removal of IIG or DBS, patient preference for IVI technique and adverse events. Results: The two groups were similar in terms of mean age $(\mathrm{P}=0.398)$, laterality $(\mathrm{P}=0.671)$, indication for treatment $(\mathrm{P}=0.175)$ and medication type $(\mathrm{P}=0.489)$. All IVI procedures were successfully completed in both groups. The mean procedure time was shorter using the IIG $(9.94 \pm 2.87$ seconds $)$ versus DBS $(21.85 \pm 7.25$ seconds $)$ technique $(\mathrm{P} \leq 0.01)$. The incidence of post-injection subconjunctival hemorrhage was higher when the DBS was applied ( $\mathrm{OR}=2.35,95 \% \mathrm{CI}=1.22-4.53)$. Patients with previous history of IVI preferred the IIG over the DBS. No other injection-related adverse events were observed in both groups.

Conclusion: DBS and IIG techniques are similarly effective and safe for the administration of intravitreal medications. The IIG appears to significantly reduce procedure time, be associated with a lower incidence of subconjunctival hemorrhage and engender better patient acceptance.

Clinical Trial Registration: ClinicalTrials.gov (NCT04455399).

Keywords: intravitreal injection, intravitreal injection guide, anti-vascular endothelial growth factor, bevacizumab, aflibercept, ranibizumab

\section{Introduction}

Intravitreal injections (IVI) have become the most commonly performed procedures in ophthalmology and are indicated for treatment of various posterior segment disorders (neovascular age-related macular degeneration [AMD], diabetic macular edema, retinal vein occlusion, choroidal neovascularization, uveitis). ${ }^{1}$ In the United States, an estimated 2 million individuals over the age of 40 years (prevalence $=1.47 \%$ ) have advanced AMD, and an estimated 1.2 million (prevalence $=1.02 \%$ ) patients have neovascular AMD in at least 1 eye; these numbers are expected to increase with the aging population. ${ }^{2}$ Early detection technology, an expanding number of indications, and the growing list of intravitreal agents will increase treatment burdens for patients and health care providers. ${ }^{3}$
Correspondence: Harvey S Uy Peregrine Eye and Laser Institute, 5/F Morning Star Center, 347 Gil Puyat Avenue, Bel Air, Makati City, I209, Philippines

Email harveyuy@yahoo.com 
To meet these logistical challenges, health care systems have employed administrative changes, streamlined IVI techniques, and IVI assistive devices. Utilizing allied health professionals for IVI administration has increased treatment capacities. $^{4}$ Novel techniques have been described that deliver faster, safer and more accurate injections. ${ }^{5-9}$ Various assistive devices may improve efficiency and patient experience. ${ }^{10-15}$ The Intravitreal Injection Guide (IIG) is a recently introduced single-use, IVI assistive instrument that features (1) a single blade lash guard; (2) a curved footplate with a distance marker; (3) a cylindrical chamber which allows controlled transscleral needle insertion, and (4) a handle. ${ }^{14,15}$ This study aimed to compare the effectiveness, procedural time, patient acceptability, and safety of the IIG versus conventional dual blade speculum (DBS) for administering IVI injections.

\section{Methods}

\section{Study Design}

We conducted a prospective, single-center, randomized clinical trial comparing the effectiveness, procedural time, patient IVI technique preference, and safety outcomes of a single-use IIG versus a dual blade speculum coupled with a surgical caliper (DBS) for administering IVI among adult patients in an ambulatory surgical center setting from November 9, 2020 to January 9, 2021. We included 200 eyes of 200 adult patients scheduled to undergo IVI for neovascular age-related macular degeneration (nAMD), polypoidal choroidal vasculopathy (PCV), diabetic macular edema (DME), retinal vein occlusion (RVO), pathologic myopia (PM) with choroidal neovascularization (CNV), and uveitic macular edema or CNV. We excluded eyes with a history of extraocular or intraocular infection within 3 months of the scheduled IVI date, scleral thinning, history of previous glaucoma surgery, history of pars plana vitrectomy, and hypersensitivity to the IVI drug, proparacaine or povidone iodine. The study was conducted in compliance with the principles of the Declaration of Helsinki. The study protocol and informed consent form were approved by the Peregrine Eye and Laser Institute Institutional Review Board (Approval\# 2020-002). All study procedures were conducted only after informed consent was obtained. The protocol has been registered with and accessible at ClinicalTrials.gov (NCT04455399).
The study eyes were randomly assigned in 1:1 fashion to one of two treatment arms: IIG and DBS. At the time of injection, a random number generator was used to generate an odd or even number for each eye. Odd eyes were assigned to IIG and even eyes assigned to DBS. All injections were performed by a single surgeon (HSU) using a disposable 30 -gauge needle attached to a $1.0 \mathrm{~mL}$ syringe. All injections were performed under topical anesthesia using $0.5 \%$ proparacaine hydrochloride ophthalmic solution (Alcaine, Alcon Laboratories, Fort Worth, TX, USA). For asepsis, 5\% povidone iodine solution (PVI) was painted on the periocular skin and eyelashes followed by instillation of 5\% PVI onto the ocular surface for at least 30 seconds.

\section{Intravitreal Injection Guide}

The IIG (Beaver-Visitec International, Waltham, MA) is a single-use, sterile, polycarbonate instrument which is Conformité Européenne (CE)-marked and United States Food and Drug Administration (US FDA) approved via 510(k) exemption. ${ }^{16}$ It was developed in the United Kingdom by Dr. Salman Waqar in collaboration with two National Health Service (NHS) trusts to help address the growing demand for innovations in intravitreal injection. ${ }^{14,15}$ Its components are (1) a lash guard to prevent needle contamination; (2) a curved triangular footplate with fixation studs for stabilization and an apex indicating placement at the limbus; (3) a cylindrical chamber guiding precise and controlled needle entry at the pars plana; and (4) a wishbone handle to ensure proper visualization of the procedure (Figure 1). It was designed to eliminate the use of a drape and speculum and to improve globe stabilization. ${ }^{14}$ Preliminary studies have reported good patient feedback with slightly less procedure pain compared to conventional IVI procedures. ${ }^{14,15}$

Administration of IVI using the IIG consisted of 4 steps: (1) simultaneous eyelid displacement and measurement of a $4 \mathrm{~mm}$ injection site posterior to the limbus by placement of the IIG footplate apex adjacent to the inferior limbal border; (2) insertion of the needle through the metal cylindrical chamber and then trans-sclerally until a stop is reached signifying a $7 \mathrm{~mm}$ intraocular needle depth; (3) drug injection by pushing the syringe plunger until a stop is reached; and, (4) removal of needle and IIG from the ocular surface (Figure 2A). 


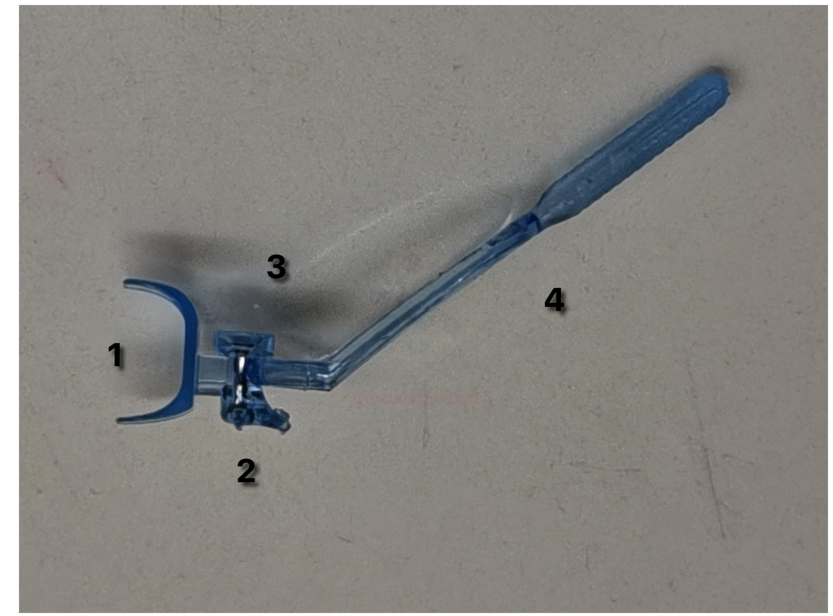

Figure I Photograph of Malosa Intravitreal Injection Guide demonstrating (I) lash guard, (2) curved triangular footplate, (3) cylindrical injection chamber, (4) wishbone handle.

\section{Dual Blade Speculum and Surgical Caliper}

Administration of IVI using the conventional DBS technique consisted of 6 steps: (1) separation of the eyelids to expose the ocular surface by application of a $13.5 \mathrm{~mm}$, sterilize titanium dual blade wire speculum (Charmant Group, Sabae-city, Fukui, Japan); (2) stabilization of the globe using a cotton tip applicator; (3) marking of the injection site using a Castroviejo surgical caliper set to $4 \mathrm{~mm}$ (E2404, Storz Ophthalmic Instruments, Bausch \& Lomb, Rochester, NY, USA); (4) trans-scleral insertion of the 30gauge needle at the injection site until at point about twothirds of the way into the eye; (5) drug injection by pushing the syringe plunger all the way; and, (6) removal of needle and DBS from the ocular surface (Figure 2B).

At the end of the injection procedure, all eyes received topical $0.5 \%$ levofloxacin instilled into the fornices. The patients were observed for about 2 minutes and queried about perfusion loss. The eyelids were then retracted to check for adverse events. The patients were then given postinjection instructions and sent home without any prophylactic antibiotic medications. IIG patients who had previously undergone IVI using DBS were queried as to which technique was preferable in terms of procedure comfort.

\section{Data Collection and Statistical Analysis}

The following data were collected: age, laterality, clinical diagnosis, type of drug injected, rate of successful IVI administration, duration of the injection procedure (in seconds), IVI technique preference, and post-injection adverse events. To determine the procedural time, a stopwatch timer was started at the moment when either the IIG or DBS device touched the eyelid and stopped when the device was removed from the ocular surface after IVI administration. Adverse events (vitreous loss, subconjunctival hemorrhage ( $\mathrm{SCH})$, perfusion loss, endophthalmitis, retinal detachment and ocular inflammation) were recorded after the injection and at the 1-month post-injection visit. If there were no cases of perfusion loss, the patients were sent home and instructed to contact the clinic in case of unexpected signs and symptoms (pain, blurred vision, eyelid swelling, diffuse redness). In case of perfusion loss, the eye would undergo paracentesis in an effort to restore perfusion. Statistical analysis was done using IBM SPSS Statistics for Mac (IBM Corporation, Armonk, NY, USA). Independent $t$-test was used to analyze means (age and time), while the chi-square analysis was done for categorical variables (presence of $\mathrm{SCH}$ and incidence of adverse events).

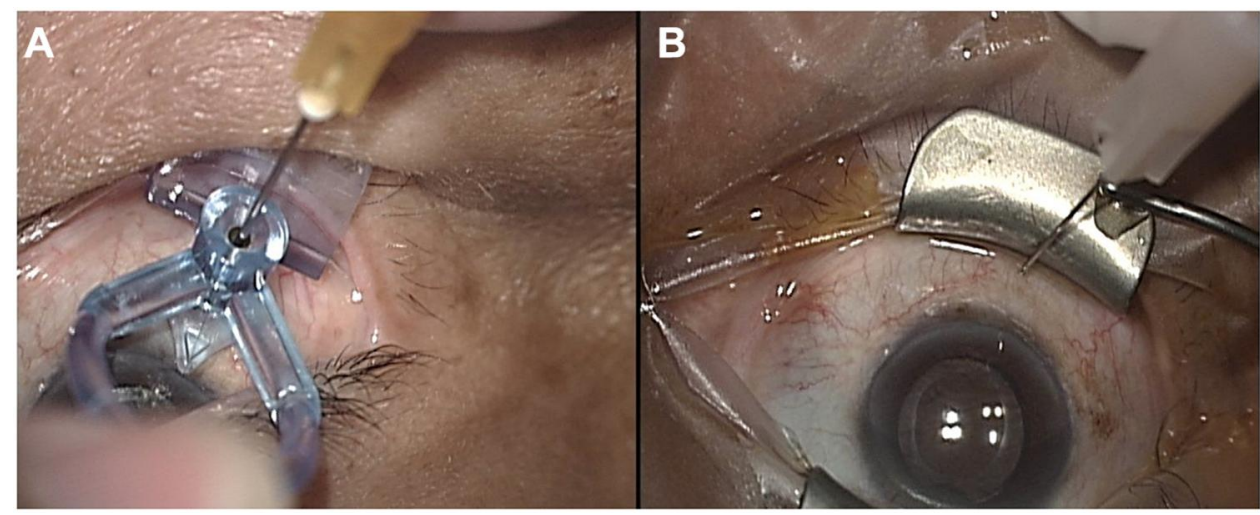

Figure 2 Operating microscope view of 30-gauge needle being inserted through: $(\mathbf{A})$ injection chamber of the Malosa Intravitreal Injection Guide with the surgical field exposed by single blade lid speculum, and (B) the pars plana with surgical field exposed by solid dual blade lid speculum. 
Table I Baseline Demographic and Clinical Characteristics

\begin{tabular}{|c|c|c|c|c|}
\hline & $\begin{array}{l}\text { Injection Guide Group } \\
(n=100)\end{array}$ & $\begin{array}{l}\text { Lid Speculum Group } \\
(n=100)\end{array}$ & $\begin{array}{l}\text { Total } \\
(n=200)\end{array}$ & p-value \\
\hline Age & $69.86 \pm 14.19$ & $70.29 \pm 13.52$ & $70.08 \pm 13.83$ & 0.398 \\
\hline Laterality, Right Eye & 49 (49\%) & $52(52 \%)$ & $101(50.5 \%)$ & 0.671 \\
\hline Indication & & & & 0.175 \\
\hline Neovascular age-related macular degeneration & $73(73 \%)$ & $73(73 \%)$ & 146 (73\%) & \\
\hline Diabetic macular edema & II (II\%) & $8(8 \%)$ & $19(9.5 \%)$ & \\
\hline Retinal vein occlusion & $4(4 \%)$ & $9(9 \%)$ & $13(6.5 \%)$ & \\
\hline Polypoidal choroidal vasculopathy & $7(7 \%)$ & $4(4 \%)$ & II (5.5\%) & \\
\hline Uveitic Macular Edema & I (I\%) & $5(5 \%)$ & $6(3 \%)$ & \\
\hline Pathologic myopia with choroidal neovascularization & $4(4 \%)$ & $\mathrm{I}(\mathrm{I} \%)$ & $5(2.5 \%)$ & \\
\hline Drug Administered & & & & 0.489 \\
\hline Bevacizumab $1.25 \mathrm{mg} / 0.05 \mathrm{~mL}$ & $52(52 \%)$ & $46(46 \%)$ & 98 (49\%) & \\
\hline Aflibercept $2.0 \mathrm{mg} / 0.05 \mathrm{~mL}$ & $35(35 \%)$ & $36(36 \%)$ & $7 \mid(35.5 \%)$ & \\
\hline Ranibizumab $0.3 \mathrm{mg} / 0.05 \mathrm{~mL}$ & II (II\%) & $12(12 \%)$ & $23(11.5 \%)$ & \\
\hline Triamcinolone $2 \mathrm{mg} / 0.05 \mathrm{~mL}$ & $2(2 \%)$ & $6(6 \%)$ & $8(4 \%)$ & \\
\hline
\end{tabular}

\section{Results}

Of the 200 eyes enrolled in this study, 100 (50\%) received IVI using the IIG and 100 (50\%) used the DBS technique. The demographic and clinical features are described in Table 1. There were no significant differences in patient age $(\mathrm{P}=0.398)$, laterality $(\mathrm{P}=0.671)$, indication for IVI $(\mathrm{P}=0.175)$ and choice of $\operatorname{drug}(\mathrm{P}=0.489)$ between the 2 treatment groups. All eyes successfully completed the injection procedure using the assigned technique. The mean procedure time was significantly shorter in the IIG group (9.94 \pm 2.87 seconds) compared to the DBS group $(21.85 \pm 7.25$ seconds $)(P \leq 0.01)$. Seventy-seven of 100 patients $(77 \%)$ who underwent IIG injection had previously undergone IVI using a DBS device. All of these patients reported less discomfort with and indicated their preference for using the IIG over the DBS.

There were no cases of perfusion loss among the 200 eyes nor were there late onset injection-related serious adverse events. The only injection-related adverse events were SCH which occurred in 52 of 200 (26\%) eyes. There was a higher incidence of post-injection $\mathrm{SCH}$ in the DBS $(34 \%)$ versus the IIG (18\%) group $(\mathrm{P}=0.01)$ (Table 2$)$. The odds of $\mathrm{SCH}$ were nearly two-and-a-half times higher when using the DBS versus the IIG technique $(\mathrm{OR}=$ $2.35,95 \% \mathrm{CI}=1.22-4.53)$.

\section{Discussion}

IVI was successfully administered in all eyes using either IIG or conventional DBS techniques. These results are in agreement with that of previous studies conducted at the United Kingdom National Health Service which reported on the effectiveness of the IIG for administering IVI while eliminating the need for draping and a separate surgical caliper. $^{14,15}$ The IIG has also been reported to provide a slightly less painful IVI experience compared to conventional techniques and generated good patient feedback. ${ }^{15}$ The post-injection survey of patients who received prior IVI using conventional DBS device demonstrated their preference for the IIG on the basis of improved comfort. Our results further demonstrate that the IIG device can shorten the procedural time and may be associated with a lower incidence of post-injection $\mathrm{SCH}$.

There currently exists a wide range of variations in performing IVI such as training qualifications of the

Table 2 Comparison of Procedure Time and Incidence of PostOperative Hemorrhage and Adverse Events

\begin{tabular}{|l|c|c|c|}
\hline & $\begin{array}{c}\text { Intravitreal } \\
\text { Injection Guide } \\
\text { Group } \\
(\mathbf{n}=\mathbf{~ 1 0 0 )}\end{array}$ & $\begin{array}{c}\text { Dual Blade } \\
\text { Lid Speculum } \\
\text { Group } \\
(\mathbf{n}=\mathbf{~ 1 0 0 )}\end{array}$ & p-value \\
\hline $\begin{array}{l}\text { Mean procedure } \\
\text { duration } \\
\text { (seconds) }\end{array}$ & $9.94 \pm 2.87$ & $21.85 \pm 7.25$ & $\leq 0.01$ \\
\hline $\begin{array}{l}\text { Subconjunctival } \\
\text { hemorrhage }\end{array}$ & $18(18 \%)$ & $34(34 \%)$ & 0.01 \\
\hline Adverse events & $0(0.0 \%)$ & $0(0 \%)$ & \\
\hline
\end{tabular}


injector, type of anesthesia, drape use, injection site, and method of ocular surface exposure. In this study, in order to isolate the effect of the assistive IVI on procedural time, the timer was activated at first contact of either IIG or DBS onto the ocular surface. This method eliminates variations in procedural time caused by routine preparation of the treatment area and focuses solely on the time consumed for the injection procedure.

The IIG is designed to facilitate the injection process. A single motion exposes the injection site, identifies the injection site and guides the needle as to the appropriate injection angle and depth. As a result, the procedure time can be shortened. As the device is disposable, the support staff also saves time by not having to sterilize instruments for succeeding patients. Time and motion studies like this are helpful for optimizing practice efficiency.

At present, 4 IVI assistive devices are commercially available: InVitria Injection Assistant (FCI Ophthalmics, Pembroke, MA), Rapid Access Vitreal Injection (RAVI) Guide (Katalyst Surgical, Chesterfield, MO); Doi-Uematsu IIG (Duckworth \& Kent Ltd., England) and the Malosa IIG. ${ }^{10-15}$ All have been demonstrated to safely assist IVI delivery. There are very few comparative studies on their clinical performance.

A recent study on patient IVI experiences revealed that of 10 steps in the process, insertion of the lid speculum was the third leading cause of discomfort following needle insertion and drape application. ${ }^{17}$ Compared to the DBS which applies pressure on both upper and lower eyelids, the single blade IIG decreases the amount of applied pressure as only one eyelid needs to be pushed away. Depending on patient-specific factors (eg small palpebral apertures, uncooperative patients), more effort may be required to apply a DBS resulting in more patient discomfort. Compared to the DBS, we found that the IIG is easier to apply as only one eyelid needs to be engaged. Theoretically, the small footprint of the IIG would facilitate its application in eyes with very small palpebral apertures. We report here successful IVI administration using the IIG in all assigned eyes. There are reports of failure to apply IVI assistive devices with relatively larger, 360 degree footprint (eg InVitria). ${ }^{13}$ Furthermore, elimination of sticky drapes and shortened procedure time may enhance patient comfort and lessen injection anxiety. All of the patients who have had prior experienced with DBSbased IVI indicated their preference for the IIG which has since become our standard of care. Comparative ease of use of assistive devices and their consistent results may aid in the employment of experienced ophthalmic nurses for IVI administration. ${ }^{4}$

No untoward side effects occurred with either IIG or DBS use in this study. The most dreaded complication of IVI is endophthalmitis, with reported incidence of $0.020 \%$ to $0.085 \% .^{18-21}$ Other IVI-related complications (retinal detachment, ocular inflammation) are rare. As of current writing, there are no existing reports of serious adverse events using the IIG. There has been a report of bleb leak and bleb-associated endophthalmitis following application of the InVitria device. ${ }^{22}$ In eyes with filtering blebs, we found that the IIG can easily be strategically placed away from the bleb in order to avoid bleb trauma.

Unexpectedly, we also observed a significantly lower incidence of $\mathrm{SCH}$ with the use of the IIG compared to the DBS. We postulate that the downward pressure by the footplate on the conjunctival surface causes the blood vessels to close off or blanch, thereby decreasing the chances of the injection needle nicking the conjunctival blood vessels. Because some patients are unable to or not willing to return to work in the presence of $\mathrm{SCH}$, a technique that reduces the incidence of SCH will enable a patient to return to work sooner and reduce income losses from absences.

As the number of IVI performed is expected to increase and pose substantial treatment burdens on health care systems worldwide, there is an unmet need for more efficient methods of IVI delivery. Potential solutions range from employing nurses to administer IVI to utilizing automated robotic systems. The relative lack of clinical trials with regards to the injection process has limited the creation and adoption of standard treatment guidelines. ${ }^{23}$

In this study, use of the IIG decreased the procedural time by half for every case. While this decrease of about 10 seconds does not seem like much, these 10 seconds consist of high value physician time. A physician or injector can essentially double the number of IVI completed in the same amount of time thereby enhancing their productivity. In an optimized set up, the support staff can prepare a series of eyes for IVI and the physician or injector can then inject these eyes rapidly, accurately and safely in an assembly line fashion with appropriate time out procedures. Using the IIG and an assembly line set up, we can typically complete injection of 4 to 5 eyes within a minute.

The cost benefit of utilizing the IIG will be highly dependent on current institutional practice. The IIG cost approximately USD 10 comparable to the cost of the InVitria device so switching from one to the other will 
generally be cost neutral. Practices currently using singleuse injection kits (inclusive of dual blade eyelid speculum, eyelid drape, cotton tip applicator, surgical caliper), costing about USD 20, will derive significant cost savings per procedure when switching to the IIG. It is difficult to quantify the cost benefit for institutions using reusable metal DBS and surgical caliper because many factors are involved in cost analysis such as the labor cost to clean, package, sterilize and store the instruments. Adopting the single-use IIG will likely lessen the risk for endophthalmitis. Irrespective of cost of goods, IIG use will likely reduce the physician procedure time. For a high volume practice, reduction of procedure time for physicians may lead to increased revenue generation as the physician will have more time to perform other functions or perform more injections per given unit of time. Lastly, we believe the turnover time will be shorter when using the IIG as there will be fewer items to prepare allowing a unit to perform more procedures per given location during the same span of time.

The main strength of this study is the adoption of a randomized clinical trial design and adherence to a defined IVI injection process that decreases confounding variables. We acknowledge the following limitations. Having a single treating investigator in this open-label study creates a potential for researcher bias, which could be decreased by involving more investigators. The short study duration and relatively small sample size in this study does not allow for detection of rare and late-onset complications. We did not take into account systemic factors that may affect $\mathrm{SCH}$ incidence. For purposes of uniformity, all treatments were administered in the inferior quadrant, so we do not know the effects of using other injection locations. Areas for future investigation include comparative studies versus other devices, inclusion of objective measurements for injection discomfort and a larger population size to detect less frequent adverse events.

Future innovations will continue to improve the efficiency and safety of IVI. In the near horizon, prefilled syringes will shorten the drug preparation step, while longer-acting drugs, sustained release or depot preparations can lessen injection frequency. In the long term, robotic assistive devices may lead to IVI automation. ${ }^{24}$

This study demonstrates that both DBS and IIG are effective means of administering IVI. Additionally, the IIG reduces procedure time, decreases the incidence of cosmetically displeasing $\mathrm{SCH}$, lessens procedural discomfort and may improve clinical efficiency and cost effectiveness of the IVI procedure. The IIG is likely to decrease patient injection anxiety and improve efficiency in practices with significant IVI volumes.

\section{Data Sharing Statement}

The data that support the findings of the study are not publicly available to protect the privacy of the study participants.

\section{Author Contributions}

Both authors made a significant contribution to the work reported, whether that is in the conception, study design, execution, acquisition of data, analysis and interpretation, or in all these areas; took part in drafting, revising or critically reviewing the article; gave final approval of the version to be published; have agreed on the journal to which the article has been submitted; and agree to be accountable for all aspects of the work.

\section{Funding}

The authors have received no funding support in the performance of this study.

\section{Disclosure}

The authors report no conflicts of interest in this work.

\section{References}

1. Lai TY, Liu S, Das S, Lam DS. Intravitreal Injection-Technique and Safety. Asia Pac J Ophthalmol. 2015;4(6):321-328. doi:10.1097/ APO.0000000000000146

2. Friedman DS, O'Colmain BJ, Munoz B, et al.; Eye Diseases Prevalence Research Group. Prevalence of age-related macular degeneration in the United States. Arch Ophthalmol. 2004;122(4):564-572.

3. Prenner JL, Halperin LS, Rycroft C, et al. Disease Burden in the Treatment of Age Related Macular Degeneration: findings From a Time-and-Motion Study. Am J Ophthalmol. 2015;160(4):725-731. doi:10.1016/j.ajo.2015.06.023

4. Michelotti MM, Abugreen S, Kelly SP, et al. Transformational change: nurses substituting for ophthalmologists for intravitreal injections - a quality-improvement report. Clin Ophthalmol. 2014;8:755-761. doi:10.2147/OPTH.S59982

5. Rahimy E, Fineman MS, Regillo CD, et al. Speculum versus Bimanual Lid Retraction during Intravitreal Injection. Ophthalmology. 2015;122 (8):1729-1730. doi:10.1016/j.ophtha.2015.02.001

6. Alattas K. Patients' tolerance of bimanual lid retraction versus a metal speculum for intravitreal injections. Clin Ophthalmol. 2016;10:1719-1721. doi:10.2147/OPTH.S113326

7. Fineman MS, Hsu J, Spirn MJ, Kaiser RS. Bimanual assisted eyelid retraction technique for intravitreal injections. Retina. 2013;33 (9):1968-1970. doi:10.1097/IAE.0b013e318287da92

8. Mason RW. Use of a Desmarres retractor for upper lid and lash isolation during intravitreal injections. Retina. 2013;33 (10):2175-2176. doi:10.1097/IAE.0b013e3182999a1b 
9. Shrier EM. Cotton-tip applicator lid retraction technique for controlled intravitreal injection. Retina. 2014;34(6):1244-1246. doi:10.1097/IAE.0000000000000219

10. Ratnarajan G, Nath R, Appaswamy S, Watson SL. Intravitreal injections using a novel conjunctival mould: a comparison with a conventional technique. Br J Ophthalmol. 2013;97(4):395-397. doi:10.1136/bjophthalmol-2012-302155

11. Han DP, McKenney KC, Kim JE, Weinberg DV, Musch DC, Singh RS. CLINICAL EVALUATION OF THE RAPID ACCESS VITREAL INJECTION GUIDE: a Handheld Instrument for Assisting Intravitreal Injections. Retina. 2017;37(4):778-781. doi:10.1097/IAE.0000000000001229

12. Watanabe K, Masafumi U, Mohamed YH, et al. Safety of Intravitreal Injection Guide. J Vitreoretin Dis. 2017;2(1):26-31. doi:10.1177/ 2474126417728622

13. Soh YQ, Chiam NPY, Tsai ASH, et al. Intravitreal injection with a conjunctival injection device: a single-center experience. Trans Vis Sci Tech. 2020;9(8):28. doi:10.1167/tvst.9.8.28

14. Waqar S. Comment on: optimisation of intravitreal injection technique using a Barraquer speculum with solid flat blade and finger stabilisation. Eye. 2019;33(3):512. doi:10.1038/s41433-018-0248-3

15. Waqar S, Ayaz A, Karamat I, et al. A novel device for rapid, safe and precise delivery of intravitreal injections. BMJ Innov. 2019;5 (1):8-12. doi:10.1136/bmjinnov-2018-000337

16. U.S. Food and Drug Administration. CFR - code of Federal Regulations Title 21. Available from: https://www.accessdata.fda. gov/scripts/cdrh/cfdocs/cfcfr/CFRSearch.cfm?fr=812.2. Accessed March 23, 2020.

17. Tailor R, Beasley R, Yang Y, Narendran N. Evaluation of patients' experiences at different stages of the intravitreal injection procedure what can be improved? Clin Ophthalmol. 2011;5:1499-1502. doi:10.2147/OPTH.S24358
18. McCannel C. Meta-analysis of endophthalmitis after intravitreal injection of anti-vascular endothelial growth factor agents: causative organisms and possible prevention strategies. Retina. 2011;31 (4):654-661. doi:10.1097/IAE.0b013e31820a67e4

19. Falavarjani KG, Nguyen QD. Adverse events and complications associated with intravitreal injection of anti-VEGF agents: a review of literature. Eye. 2013;27(7):787-794. doi:10.1038/eye.2013.107

20. Haddock LJ, Ramsey DJ, Young LH. Complications of subspecialty ophthalmic care: endophthalmitis after intravitreal injections of anti-vascular endothelial growth factor medications. Semin Ophthalmol. 2014;29(5-6):257-262. doi:10.3109/ 08820538.2014 .959616

21. Sigford DK, Reddy S, Mollineaux C, et al. Global reported endophthalmitis risk following intravitreal injections of anti-VEGF: a literature review and analysis. Clin Ophthalmol. 2015;9:773-781. doi:10.2147/OPTH.S77067

22. Baneke AJ, Vakros G, Sharma V, Wong SC. Bleb-related endophthalmitis after use of the InVitria injection guide. Retin Cases Brief Rep. 2020;14(1):33-34. doi:10.1097/ICB.0000000000000631

23. Grzybowski A, Told R, Sacu S, et al.. 2018 update on intravitreal injections: euretina expert consensus recommendations. Ophthalmologica. 2018;239(4):181-193. doi:10.1159/000486145.

24. Ullrich F, Michels S, Lehmann D, Pieters RS, Becker M, Nelson BJ. Assistive device for efficient intravitreal injections. Ophthalmic Surg Lasers Imaging Retina. 2016;47(8):752-762. doi:10.3928/2325816020160808-09
Clinical Ophthalmology

\section{Publish your work in this journal}

Clinical Ophthalmology is an international, peer-reviewed journal covering all subspecialties within ophthalmology. Key topics include: Optometry; Visual science; Pharmacology and drug therapy in eye diseases; Basic Sciences; Primary and Secondary eye care; Patient Safety and Quality of Care Improvements. This journal is indexed on PubMed
Dovepress

Central and CAS, and is the official journal of The Society of Clinical Ophthalmology (SCO). The manuscript management system is completely online and includes a very quick and fair peer-review system, which is all easy to use. Visit http://www.dovepress.com/ testimonials.php to read real quotes from published authors. 